\title{
LAS NUEVAS TECNOLOGÍAS EN EL APRENDIZAJE CONSTRUCTIVO
}

\author{
Miguel Ángel Herrera Batista \\ Universidad Autónoma Metropolitana, México
}

\section{INTRODUCCIÓN}

Uno de los grandes retos en la actualidad es mejorar la calidad de la educación. Procurar que los estudiantes aprendan más y de mejor forma es la preocupación de docentes e instituciones educativas. Para ello es necesario que los alumnos cuenten con ambientes de aprendizaje más efectivos y didácticos; entornos educativos que les permitan desarrollar sus habilidades para pensar y su capacidad para aprender. En este sentido, los ambientes virtuales de aprendizaje representan nuevas posibilidades de acceso a la educación. Sin embargo, cuando el diseño de dichos ambientes se realiza sin un sustento científico adecuado y sin una propuesta didáctica claramente definida, los beneficios de las actividades de aprendizaje pueden verse disminuidos notablemente. No es difícil encontrar ambientes virtuales que sólo se limitan a presentar información ${ }^{1} \sin$ una planeación apropiada de la instrucción, es decir, sin un diseño instruccional ${ }^{2}$ idóneo, que considere los factores psicológicos del aprendizaje y el papel que juegan las NT en ese proceso.

De acuerdo con las teorías actuales ${ }^{3}$ de la psicología cognitiva, la información por sí misma no propicia conocimiento, es necesario proveer una serie de condiciones que favorezcan el proceso de aprendizaje. No se trata simplemente de entregar información para que el aprendizaje se produzca. Es necesario propiciar las "transacciones didácticas fundamentales que se presentan entre docentes y estudiantes o estudiantes entre sí, y que contribuyen a la circularidad ${ }^{4}$ comunicativa indiscutible en la construcción de los saberes", Fainholc (1999, pp. 64-65).

\footnotetext{
${ }^{1}$ En una muestra de 25 sitios educativos iberoamericanos analizados (mexicanos, españoles y argentinos principalmente), se encontró que un alto porcentaje (más del 70\%) sólo proporcionaba información y una dirección de correo electrónico para interactuar. Cabe aclarar que, a la muestra revisada por el autor, se suman otros sitios revisados por Susana Badillo, Selene Martínez y Patricia García, estudiantes de la Especialización en Diseño de la Universidad Autónoma Metropolitana Azcapotzalco, quienes encontraron una situación similar. En este sentido consideramos que la entrega de información y la interacción mediante correo electrónico no son suficientes. Para procurar un mejor nivel de aprendizaje se requieren actividades que propicien el procesamiento de la información mediante la acción cognitiva por parte del estudiante.

${ }^{2}$ La educación como actividad prescriptiva, aspira a una pretensión de la realidad, un diseño anticipado de lo que se espera conseguir. Es por ello que la planeación de la educación abarca niveles y contextos muy diversos. Cuando la planeación se enfoca específicamente en la práctica del acto educativo, suele hablarse del diseño de la práctica docente, diseño de la instrucción o simplemente diseño instruccional. Dicho concepto se enmarca dentro del enfoque didáctico de la tecnología educacional, que tiene por objeto hacer más eficiente la enseñanza a través de la aplicación de principios científicos y el uso de recursos tecnológicos. Briggs y Wager (1981), se refieren al diseño instruccional como un sistema para resolver problemas, en donde lo primero es definir el problema, que en este caso es determinar cuál es la mejor forma de impartir la enseñanza; Anglin (1995), afirma que el diseño instruccional es un sistema que busca aplicar los principios derivados de la ciencia para la planeación, diseño, creación, implementación y evaluación efectiva y eficiente de la instrucción; Rogers (2002) señala que el diseño instruccional se ocupa del proceso de enseñanzzaprendizaje pero de una manera más formal y sistemática, que la practica docente común y agrega que, el análisis sistemático de cada componente del proceso diseñado contribuye a alcanzar el éxito. Aunque existen diferencias el enfoque que se da al diseño instruccional, en general se piensa que consiste en llevar los avances científicos y tecnológicos a la práctica educativa. En este trabajo asumimos dicha postura.

${ }^{3}$ Los nuevos paradigmas establecen que, para que pueda darse el aprendizaje, es necesario realizar acciones cognitivas que modifiquen las estructuras intelectuales del individuo, por lo que la recepción de la información no constituye en sí, ningún aprendizaje cognitivo. Este tema será tratado ampliamente en el primer capítulo de este trabajo.

${ }^{4}$ Por circularidad, Fainholc se refiere al diálogo que se establece entre los participantes en una situación de aprendizaje, en donde el sentido de la comunicación es bidireccional y no unidireccional.
} 
Así, en torno al problema puede advertirse que:

- La creciente demanda de ambientes virtuales de aprendizaje propicia una acelerada oferta de espacios educativos en línea.

- Una parte importante de dichos espacios se limita a presentar información sin un manejo estratégico de ésta y sin un diseño apropiado de actividades de aprendizaje.

- Los nuevos recursos tecnológicos deben ser utilizados para enriquecer la oferta educativa y no sólo en términos cuantitativos sino, ante todo, en términos cualitativos.

La atención al problema involucra la participación interdisciplinaria de la psicología cognitiva, las teorías instruccionales y del diseño entre otras. Nemirovski, Neuhaus y Schlageter (1998) señalan que existen tres tipos de requerimientos necesarios en el diseño de ambientes virtuales educativos: los requerimientos de dominio, referidos a los contenidos propios de la asignatura, en donde la selección, organización y la secuenciación de los contenidos constituyen una tarea fundamental del proceso ${ }^{5}$; los requerimientos psicopedagógicos, que corresponden al enfoque teórico y práctico de la educación en general y del aprendizaje en particular de acuerdo con los paradigmas y las estrategias didácticas asumidas; los requerimientos de interfaz, los que se derivan de las características propias del medio y el nivel de interactividad que serán utilizados.

La presente investigación está centrada en los factores educativos, con el porosito de explicar cómo se insertan las NT en los procesos de aprendizaje y cómo pueden ser utilizadas dichas tecnologías de manera más efectiva a favor de mejores ambientes virtuales. Se proponen cinco estrategias didácticas. Partiendo de ello se realizó una investigación de campo con el propósito de verificar que: el uso de estrategias didácticas sustentadas en las teorías cognitivas contribuye de manera sustancial en el diseño de ambientes virtuales didácticos. La experiencia obtenida permitió observar un avance significativo en los puntajes obtenidos por una muestra aleatoria de estudiantes antes y después de interactuar en un ambiente virtual diseñado con base en el modelo propuesto.

\section{UNA APROXIMACIÓN AL CONCEPTO DE NUEVAS TECNOLOGÍAS EN LA EDUCACIÓN}

El concepto genérico de Nuevas Tecnologías puede resultar ambiguo, sin embargo, es importante, desde nuestra perspectiva, acotar el concepto. Cabero (2000, p.17) considera que una de las características que distinguen a las NT es que "giran en torno a cuatro medios básicos: la informática, la microelectrónica, los multimedia y las telecomunicaciones". En ese sentido, para los propósitos de esta investigación, entendemos por Nuevas Tecnologías, todos los medios desarrollados en torno al surgimiento de la ciencias de la Informática y que permiten la comunicación e interacción con fines educativos; de manera sincrónica o asincrónica; de forma individual o colectiva; que utilizan la computadora como principal medio de comunicación e interacción entre los sujetos del acto educativo y; que permiten acceder a recursos y servicios desde computadoras

\footnotetext{
${ }^{5} \mathrm{Si}$ bien es cierto que durante años se consideró una actividad delegada casi exclusivamente a los especialistas, en la actualidad se pretende que el docente, basado en la experiencia previa, participe de manera activa en el diseño instruccional y realice aportaciones para enriquecer el proceso. Los nuevos paradigmas proponen la figura del docente investigador y
} 
distantes. Dentro de esta categoría se encuentran: el hipertexto, los multimedia, Internet, grupos de discusión, video-enlaces, correo electrónico, las charlas sincrónicas o chats, entre otros.

La gran capacidad y flexibilidad de las NT para la comunicación y la interacción destacan su función formativa "por cuanto apoyan la presentación de determinados contenidos, lo que puede ayudar a guiar, facilitar y organizar la acción didáctica, así como condicionan el tipo de aprendizaje a obtener, ya que pueden promover diferentes acciones mentales en los alumnos" (Cabero, 2000, p. 144). Sin embargo, anque se acepta que las nuevas tecnologías poseen un alto potencial en la enseñanza, es necesario analizar las funciones cognitivas en las que impactan dichas tecnologías para tratar de definir qué papel juegan las NT en los procesos de aprendizaje. Una mejor comprensión de ello permitirá un diseño más apropiado en los ambientes virtuales de aprendizaje.

\section{3. $\quad$ LAS NT Y LOS PROCESOS COGNITIVOS DEL APRENDIZAJE}

Desde una óptica cognitivista ${ }^{6}$ el aprendizaje se concibe como un cambio en las estructuras mentales del hombre. Dichas estructuras corresponden a modelos creados a través de la experiencia individual y tienen como base el conocimiento previo. Cada concepto se encuentra ligado a una serie de atributos que lo distinguen y que están previamente definidos en las estructuras cognitivas. Todas esas características en su conjunto, así como acciones relacionadas con éstos pueden ser evocadas por el concepto que define cierto objeto. Cada grupo de conceptos se construye ${ }^{7}$ como resultado de la experiencia del individuo a través de su interacción con el medio natural y social. Desde esta perspectiva, las estructuras mentales no son inmutables, sino que van cambiando y haciéndose más complejas a través de las experiencias de aprendizaje. Cuando este proceso de cambio es producido por la experiencia ${ }^{8}$, y es más o menos permanente, entonces se define como aprendizaje.

\subsection{Los procesos de adquisición del conocimiento según Piaget}

Gran parte de lo que hoy se sabe con respecto al aprendizaje y los procesos de adquisición del conocimiento se sustenta en las aportaciones de Piaget. Como lo señala Ferreiro (1999, p. 84) "Piaget se ocupa de la parte nuclear, medular de los procesos de aprendizaje: los procesos de adquisición de conocimientos." Piaget desarrolla toda su teoría teniendo como objeto de estudio la inteligencia humana y su función adaptativa. Considera que la inteligencia se manifiesta a través de la constante adaptación de los esquemas mentales del individuo a su ambiente. Dicha adaptación conduce al desarrollo cognitivo y al aprendizaje. Piaget considera que el aprendizaje es motivado por un proceso denominado equilibración, que definió como una tendencia natural e innata del individuo para modificar sus esquemas mentales y dar coherencia al entorno percibido. El proceso de equilibración desencadena a su vez otros dos procesos complementarios entre sí: los procesos de asimilación y acomodación.

\footnotetext{
enriquecedor de su práctica educativa en lugar de la figura pasiva del docente como simple ejecutor del currículo.

${ }^{6}$ En la literatura especializada se usa de manera indistinta los términos cognitivista o cognoscitivista para referirse a una teoría específica en la que se explica el aprendizaje como cambios en las estructuras de pensamiento y no sólo en la conducta observable como en la teoría conductista.

${ }^{7}$ Hacemos referencia a la orientación constructivista del aprendizaje, la cual sostiene que el conocimiento no es una copia fiel de la realidad sino una representación construida por el individuo, idea que se apoya en las teorías de Piaget, Vygotski y

Ausubel, así como en el Modelo de Procesamiento Humano de la Información, entre otras.
} 
Específicamente Piaget considera que la inteligencia tiene una doble función: la adaptación y la organización. La adaptación se refiere al proceso a través del cual se adquiere el equilibrio entre la asimilación y la acomodación. La organización es la función que permite ordenar y reestructurar la información en los elementos internos de la inteligencia (esquemas y estructuras). "Hay, por lo tanto, dos formas diferentes de actividad: una, el proceso de entrada de la información (adaptación); otra, el proceso de su estructuración (organización).”(Araújo, 1993, p. 67)

\subsection{La interacción social y su importancia en el aprendizaje: Vygotski}

Para Vygotski, el entorno social representa un papel fundamental en el aprendizaje. De acuerdo con él, el hombre no es un simple receptor de estímulos, sino que actúa sobre éstos, transformándolos. Esta transformación es posible gracias a la acción mediadora de dos tipos de instrumentos: las herramientas y los signos. "Los mediadores son instrumentos que transforman la realidad en lugar de imitarla. Su función no es adaptarse pasivamente a las condiciones ambientales sino modificarlas activamente", Pozo (1994, p. 195). De acuerdo con Vygotski, la diferencia sustancial entre las herramientas y los signos es, que las primeras actúan sobre el estímulo transformándolo, mientras que los signos, a quién transforman es al sujeto. Los signos están asociados en sistemas los cuales se encuentran vinculados a unidades de significados. Estos significados son producidos, primero en el entorno social y posteriormente son adoptados por el individuo. Sin embargo, en la adquisición de los signos no basta tomarlos del entorno social; se requiere interiorizarlos. De acuerdo con Vygotski el proceso de aprendizaje se da en dos etapas: en la primera el aprendiz adopta los significados del contexto social; en la segunda, los significados son interiorizados.

Otro aspecto central en la teoría de Vygotski es el concepto denominado zona de desarrollo próximo (ZDP). De acuerdo con esta teoría, existe una diferencia entre lo que un individuo es capaz de hacer de manera autónoma (desarrollo efectivo) y lo que es capaz de hacer con la guía de otros individuos (desarrollo potencial). Ello destaca la importancia del facilitador en el proceso de aprendizaje, pues las funciones superiores se generan a partir de las relaciones entre los individuos. Como puede observarse, para Vygotski, en el proceso de adquisición de instrumentos mediadores o significados, la interacción social es fundamental

\section{PRINCIPALES FUnCIONES DE LAS NT EN EL PROCESO DE APRENDIZAJE \\ El análisis y la reflexión sobre los procesos cognitivos involucrados en el aprendizaje nos} conducen a distinguir dos funciones específicas de las NT en el proceso:

- Provisión de estímulos sensoriales

- Mediación cognitiva

Las cuales se definen a continuación.

\footnotetext{
${ }^{8}$ En donde el procesamiento interno de la información propicia los cambios cognitivos y "no son producto del desarrollo físico o enfermedad" como lo señala Woolfolk, (1993, p.173).
} 


\subsection{Las NT en la provisión de estímulos sensoriales}

La provisión de estímulos sensoriales es una de las funciones básicas de las NT en la generación de aprendizajes. En el proceso de aprendizaje las NT ofrecen estímulos de entrada los cuales son decodificados por el aprendiz.

Para comprender mejor su función en la provisión de estímulos sensoriales, nos apoyaremos en el modelo del Procesamiento Humano de la Información (PHI). Este modelo "fue desarrollado por Newell y Simon en 1972 y revisado por el último en 1978" (Castañeda y López, 1995, p. 29). El modelo se basa en la analogía mente-computadora, en la cual, ambos sistemas reciben, procesan, almacenan y recuperan información. Propone la existencia de un sistema de almacenamiento de información conformado por una memoria a largo plazo, una memoria a corto plazo y una memoria de sensorial. De acuerdo con dicho modelo, la mente humana recibe información, la procesa, la almacena y genera respuesta. El proceso se inicia a través del registro sensorial de los estímulos provenientes del medio ambiente. Esta información es seleccionada y filtrada a través de mecanismos de atención y percepción. Así, sólo una parte de la información es codificada y conducida hasta la memoria a corto plazo. "Ahí, su almacenamiento es limitado y corto; la información se usa y se pierde, a menos que se ensaye. La información que se retiene para ser recordada posteriormente, se conecta con los conocimientos previamente existentes y se codifica en la memoria a largo plazo, que es un almacenamiento aparentemente permanente."(Woolfolk, 1995, p. 247).

Los elementos que definen este modelo se pueden representar en el siguiente gráfico:

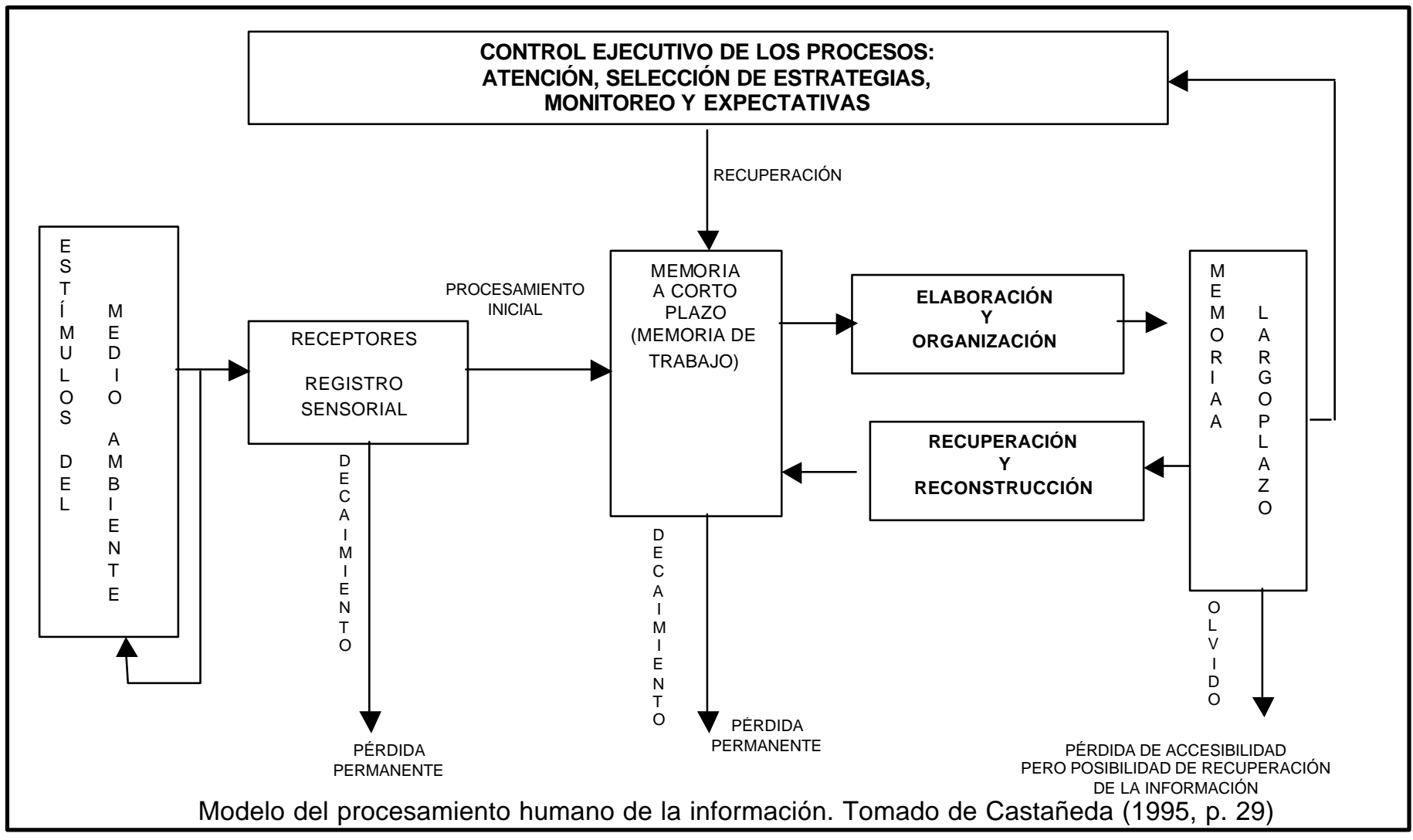

Siguiendo este modelo, las NT pueden ubicarse en la parte de estímulos del medio ambiente. Es evidente que, cuando las NT son utilizadas como medio de interacción y comunicación en 
actividades de aprendizaje, su función es, en esencia, la provisión de estímulos sensoriales. Las posibilidades de las NT para estimular los sentidos, son cada vez mayores, por lo que su potencialidad en tareas educativas no tiene precedente en la historia de los avances tecnológicos.

\subsection{Las NT como interfaz en la provisión de estímulos sensoriales}

En términos generales se puede decir que una interfaz es un objeto o recurso material o inmaterial que media entre dos entidades que interactúan y que puede ser el usuario y el escenario sobre el que actúa. Mercovich", señala que "Cuando uno usa una herramienta, o accede e interactúa con un sistema, suele haber "algo" entre uno mismo y el objeto de la interacción. Ese "algo", que es a la vez un límite y un espacio común entre ambas partes, es la interfaz."

En este sentido, en un ambiente virtual de aprendizaje, la interfaz actúa directamente en el ámbito sensorial dando forma y dirección a una de las funciones básicas de las NT en el aprendizaje: la previsión de estímulos sensoriales.

En términos generales las NT pueden ser utilizadas en la generación de estímulos de diversa índole: estímulos visuales, utilizados fundamentalmente para transmitir información a través de texto e imágenes utilizando códigos verbales (a través del lenguaje escrito) y no verbales (a través del manejo de los atributos visuales como el color, la textura, etc.); estímulos auditivos, a través de sonidos diversos y voz humana; estímulos táctiles, a través del órgano de la piel mediante el uso de sensores principalmente; estímulos olfativos, se sabe que se han realizado intentos por sintetizar olores de manera virtual, lo cual podría ser importante para algunos casos en el aprendizaje, sobre todo en la simulación; estímulos gustativos, aunque quizá éste sea uno de los sentidos más relegado por las NT no debe sorprendernos que en poco tiempo aparezcan los primeros resultados de la incorporación de estos estímulos en el uso de la computadora.

Uno de los aspectos importantes a destacar de las NT como proveedoras de estímulos sensoriales, es que la información recibida debe ser debidamente codificada, para poder ser utilizada con fines educativos. En este sentido se debe tener presente que la percepción visual y auditiva están vinculadas a la comunicación verbal, que constituye un lenguaje altamente estructurado. Por otro lado, todos los sentidos en su conjunto pueden servir para comunicar emociones y crear condiciones ambientales favorables o desfavorables para el aprendizaje.

La previsión de estímulos sensoriales tiene al menos dos dimensiones:

Su capacidad atencional. Se refiere a la potencialidad que tiene la interfaz para centrar la atención del aprendiz en los estímulos relevantes. Esta potencialidad puede manifestarse a través de dos formas: enfatizando los aspectos relevantes de la información o, inhibiendo los ruidos e interferencias del entorno.

Su capacidad motivadora. Se refiere al uso de recursos que pueden utilizarse para motivar al aprendiz en su tarea.

\footnotetext{
9 Mercovich, Eduardo. Ponencia sobre Diseño de Interfaces y Usabilidad: cómo hacer productos más útiles, eficientes y seductores. La intersección entre factores humanos, diseño gráfico, interacción y comunicación (en línea) http://planeta.gaiasur.com.ar/infoteca/diseno-de-interfaces-y-usabilidad.html. Citado por Buitrón, p. 48.
} 


\subsubsection{Las NT y los procesos de atención}

Para entender los mecanismos de los procesos atencionales algunos investigadores desarrollaron modelos de atención. Algunos de esos modelos son conocidos genéricamente como modelos de filtro, los cuales asumen la existencia de una estructura central que no puede procesar más de un mensaje por unidad de tiempo. "Para proteger este mecanismo de <<sobrecargas >> de información, inevitablemente debía existir el correspondiente cuello de botella o filtro que regulaba la entrada de información..." (De Vega, 1993, p. 144). Los paradigmas actuales se basan en un modelo de atención limitada que supone la existencia de "políticas de distribución" de la atención de manera que se regula de acuerdo a la tarea o tareas a realizar, así como a los estímulos que actúan sobre la atención involuntaria. Esta política de distribución depende de diversos aspectos como: Las disposiciones duraderas, que rigen la atención involuntaria o automática cuando se presenta un estímulo nuevo (por ejemplo un ruido inesperado); las intenciones momentáneas, que dirigen los recursos atencionales selectivamente a ciertos estímulos (como escuchar cierto timbre de voz); la evaluación de demandas, establece el consumo de recursos atencionales en cada actividad. Algunas tareas no demandan demasiados recursos mientras que otras exigen gran parte.

Lo anterior muestra la complejidad de los procesos de atención y la cantidad de factores que intervienen en dichos procesos, lo importante es que se tenga presente que la atención es un recurso limitado. Con relación a lo anterior, es importante que en los ambientes virtuales de aprendizaje se administren los recursos atencionales de manera adecuada. No es conveniente sobrecargar o saturar de información la interfaz. Con frecuencia, el abuso en animaciones, colores, texturas, textos, sonidos, etc., prácticamente bombardean de información al usuario sin una jerarquía que permita discriminar los elementos superfluos de los realmente importantes. Es posible que en casos específicos se requiera de mayores cantidades de información, en dichos casos existe la posibilidad de utilizar formatos especiales para "bajar" la información o imprimirla. Otro aspecto a considerar es que cada estudiante o usuario del ambiente virtual posee diferencias individuales importantes. En importante no diseñar para un usuario estándar sino para la gran diversidad de estilos cognitivos.

\subsubsection{Las NT y la motivación al aprendizaje}

La importancia de la motivación en el aprendizaje es fundamental. Se dice que la motivación es un impulso a la acción y para la obtención de resultados. López, Castañeda y Almaguer (1993, p.50), señalan que "Al tratar el tema de la motivación es interesante establecer una distinción entre lo extrínsecoo intrínseco de ella, en el sentido de que los satisfactores que se desean lograr guardan o no relación con lo que se hace para obtenerlos". La motivación intrínseca se pone de manifiesto cuando el aprendiz tiene un verdadero interés por aprender y comprender los conceptos estudiados y no sólo por pasar un examen, acreditar la materia, u obtener otro tipo de beneficios externos. Betancourt (2001, p.58) destaca que "Los estudiantes se comprometen en las actividades docentes intrínsicamente motivadas, porque traen consigo cierto tipo de estado interno que ellos encuentran reconfortante, no por alcanzar una recompensa externa..." La motivación extrínseca se refiere a los estímulos agradables que el estudiante recibe del entorno y que puede consistir en premios adicionales, el reconocimiento del entorno social o, simplemente sensaciones placenteras. Una diferencia importante entre la motivación intrínseca y la extrínseca es que en esta última la motivación 
puede desaparecer al momento de conseguir la recompensa buscada, mientras que en la intrínseca la motivación tiende a ser permanente. "El grado de motivación intrínseca varía positivamente con respecto a la magnitud de las recompensas intrínsecas en la tarea" Betancourt (2001, pp. 59-62).

Otro tipo de motivación es la llamada motivación defensiva. De acuerdo con este tipo de motivación, los estudiantes muy ansiosos o bajo condiciones de prueba altamente estresantes, obtienen un rendimiento más bajo en el aprendizaje, mientras que los estudiantes con poco nivel de ansiedad o en condiciones no estresantes obtienen mejores niveles de rendimiento y aprendizaje.

La motivación por logro, es "definida como la motivación por tener éxito, por ser bueno en algo" (Gagné y Berliner, 1988, en Arancibia 1999, p. 195). Es importante que se impongan retos superables para los alumnos ya que de lo contrario es posible que se sientan frustrados y pierdan el interés por aprender. Keller (en Khan, 1998, pp. 96-98), desarrolló un modelo motivacional en 1993. De acuerdo con su modelo, la motivación puede clasificarse en cuatro categorías: interés, relevancia, expectativas y satisfacción. El interés se refiere a mantener y manejar la curiosidad del aprendiz; la relevancia se refiere a relacionar la situación de aprendizaje con las necesidades y motivos del aprendiz; las expectativas se refieren a las aspiraciones individuales del aprendiz; la satisfacción se refiere a la motivación continua para seguir trabajando por conseguir metas similares.

Sin pretender agotar el tema, se han presentado algunas de las clasificaciones más conocidas en el ámbito educativo. Se puede observar que, mientras la clasificación extrínseca intrínseca enfatiza la importancia de los factores externos e internos; la motivación por logro y la defensiva, así como el modelo de Keller tienen relevancia en la planeación de las actividades y el diseño instruccional ${ }^{10}$.

Con relación al tema que nos ocupa, se pueden distinguir tres ejes sobre los que se puede trabajar la motivación para el aprendizaje:

- Las NT y la provisión de estímulos sensoriales.

- El diseño instruccional y la planeación de las actividades.

- La operación y el manejo de la comunicación en el curso.

Con respecto a las NT y la provisión de estímulos sensoriales, un primer paso es cerciorarse de que las imágenes, textos, sonidos y demás elementos de la interfaz, sean correctamente recibidos por los estudiantes. Una imagen difusa puede inhibir o desalentar la participación del estudiante. Además el uso de imágenes, animaciones o temas deben tener un significado para el estudiante. Algunos autores aseguran que el uso de la computadora en tareas educativas es un incentivo en sí mismo para la mayoría de los estudiantes, sin embargo es importante tener en cuenta también que, durante el trabajo en línea el estudiante podrá tener acceso a otros ambientes o sitios Web que despierten más su interés. En este sentido, la variación de estímulo y de canal sensorial es un medio potencialmente adecuado para la motivación.

\footnotetext{
${ }^{10}$ El tema de diseño instruccional será abordado más adelante, pero por ahora diremos que se refiere a la planeación del proceso educativo, en especial al diseño de actividades de aprendizaje.
} 
Con relación al diseño instruccional y la planeación de las actividades de aprendizaje, se debe tener cuidado que éstas impliquen retos superables pero al mismo tiempo que representen un desafío para el estudiante.

Por último, con relación a la operación y al manejo de la comunicación en un curso, en general será muy importante que todo mensaje del estudiante reciba una respuesta adecuada en tiempos y formas, dependiendo, desde luego del tipo de programa educativo, sobre todo cuando es a distancia. La educación en esta modalidad suele presentar más altos niveles de deserción ${ }^{11}$. Aunque existen muchos factores (como las características del programa educativ, el interés personal, las condiciones sociales, etc.), en muchas ocasiones, la deserción puede ser motivada por una sensación de separación (más psicológica que física) entre el estudiante y el grupo o la clase en virtud de que no recibe respuesta a sus dudas e inquietudes. Es decir, la falta de retroalimentación por parte del cuerpo docente o de otros compañeros puede ser un factor que desmotive la participación del estudiante, sin embargo, para sentirse separado del grupo no sólo cuenta la respuesta a sus dudas propiamente dichas, sino también la exclusión de las actividades académicas o la una deficiente comunicación con los demás participantes. Cuando un alumno siente que no es tomado en cuenta, puede disminuir notablemente su interés en el curso.

\subsection{Las NT: mediación cognitiva en la generación del aprendizaje}

Además de la provisión de estímulos sensoriales, otra de las funciones de las NT en la generación del aprendizaje es la mediación cognitiva, que permite el intercambio entre las estructuras mentales propias del aprendiz y las estructuras mentales ajenas a él o hechos observables que son interpretados por él.

Para explicar el papel de las NT en la generación del conocimiento, nos apoyaremos en las aportaciones de Gagné a la teoría del aprendizaje. Además de su conocida propuesta sobre los tipos de aprendizaje, Gagné, señala tres elementos fundamentales en el proceso de aprendizaje: los factores externos, los factores internos y los procesos internos que dan forma al suceso del aprendizaje. Los factores externos son la contigüidad, la repetición y el refuerzo ${ }^{12}$. El refuerzo se refiere a estimular el aprendizaje produciendo un estado de satisfacción ante una respuesta esperada. Los factores internos son: la información relevante, las habilidades intelectuales y las estrategias cognitivas. La información relevante puede provenir de la memoria (aprendizajes previos anteriores o inmediatos) o puede ser dada directamente al aprendiz. Las habilidades intelectuales son necesarias para adquirir nuevos conocimientos; el aprendiz debe ser capaz de manipular símbolos lingüísticos y códigos. Las estrategias cognitivas son genéricas y pueden aplicarse a diversidad de situaciones.

\footnotetext{
${ }^{11}$ Cornell y Martin (en Khan, B., et. al., 1998, pp. 93-94) señalan que entre el 30 y el $50 \%$ de los estudiantes que inician un programa de educación a distancia no concluyen sus estudios.

${ }_{12}^{2}$ La contigüidad es un principio de aprendizaje según el cual, el tiempo entre un estímulo y la respuesta debe ser mínimo. La repetición se refiere al número de ensayos necesarios para el aprendizaje. Aquí Gagné muestra claramente su influencia conductista, sin embargo, cuando considera los aspectos internos como las habilidades y estrategias intelectuales, muestra su postura cognitivista. En realidad Gagné asume una perspectiva ecléctica.
} 
Gráficamente:

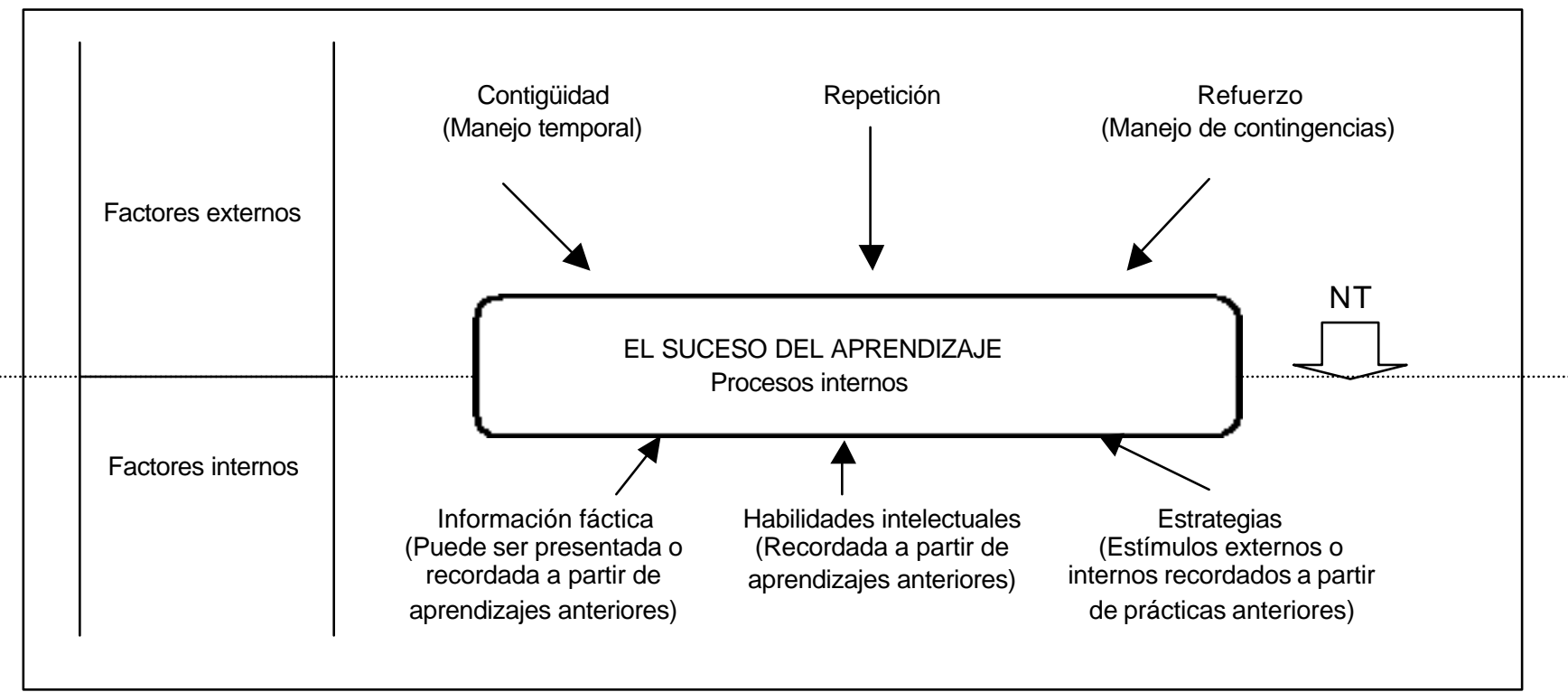

Figura 1: Factores externos e internos del aprendizaje según Gagné. Adaptado de Araújo (1993, p.49)

Para que el aprendizaje tenga lugar, es necesario que la relación entre los factores externos y los factores internos se lleve a cabo. Esta relación se realiza a través de una línea que separa ambos tipos de factores. Dicha línea es el campo de acción de las NT en la generación del aprendizaje.

Es importante entender que no nos referimos a la provisión de estímulos sensoriales (relacionada principalmente con la interfaz gráfica), sino al papel que juegan las NT como puente de enlace entre dos o más estructuras mentales involucradas en el proceso de aprendizaje, es decir, nos referimos a la mediación cognitiva. Es a través de esta mediación cognitiva que el aprendizaje puede tener lugar. Mialaret (2001), destaca la importancia de las relaciones psicológicas entre los participantes en una situación general de aprendizaje. Para nosotros, cuando la situación de aprendizaje es dada a través de ambientes virtuales, las NT son el conducto para a mediación cognitiva que constituye la esencia del aprendizaje. Cabe aclarar, sin embargo, que la mediación cognitiva va más allá de lo definido por Mialaret. Consideramos que la mediación no sólo puede ocurrir entre el aprendiz y uno o más individuos, sino también en la relación del aprendiz con el entorno observado, así como la relación interna aprendiz-consigo mismo que puede ocurrir en el aprendizaje. Por otro lado, la mediación cognitiva puede ser unidireccional o multidireccional. Es unidireccional cuando el aprendiz tiene acceso a una fuente de información pero no tiene posibilidad de ser retroalimentado por los autores o tutores. Es multidireccional cuando el aprendiz puede recibir información y ser retroalimentado por un miembro de la comunidad de aprendizaje ${ }^{13}$ como lo muestra la siguiente figura.

\footnotetext{
${ }^{13}$ En este sentido, la comunidad de aprendizaje está constituida por las estructuras mentales que interactúan, en donde se incluye el diálogo que el estudiante establece consigo mismo al modificar sus estructuras mentales. Como lo señala González (2003, p. 11), "A pesar de qué en muchas escuelas se ofrece un currículo rígido y lineal, el estudiante, en su afán por construir su propia estructura de conocimientos y significados, avanza eligiendo bloques de acuerdo a sus necesidades."
} 


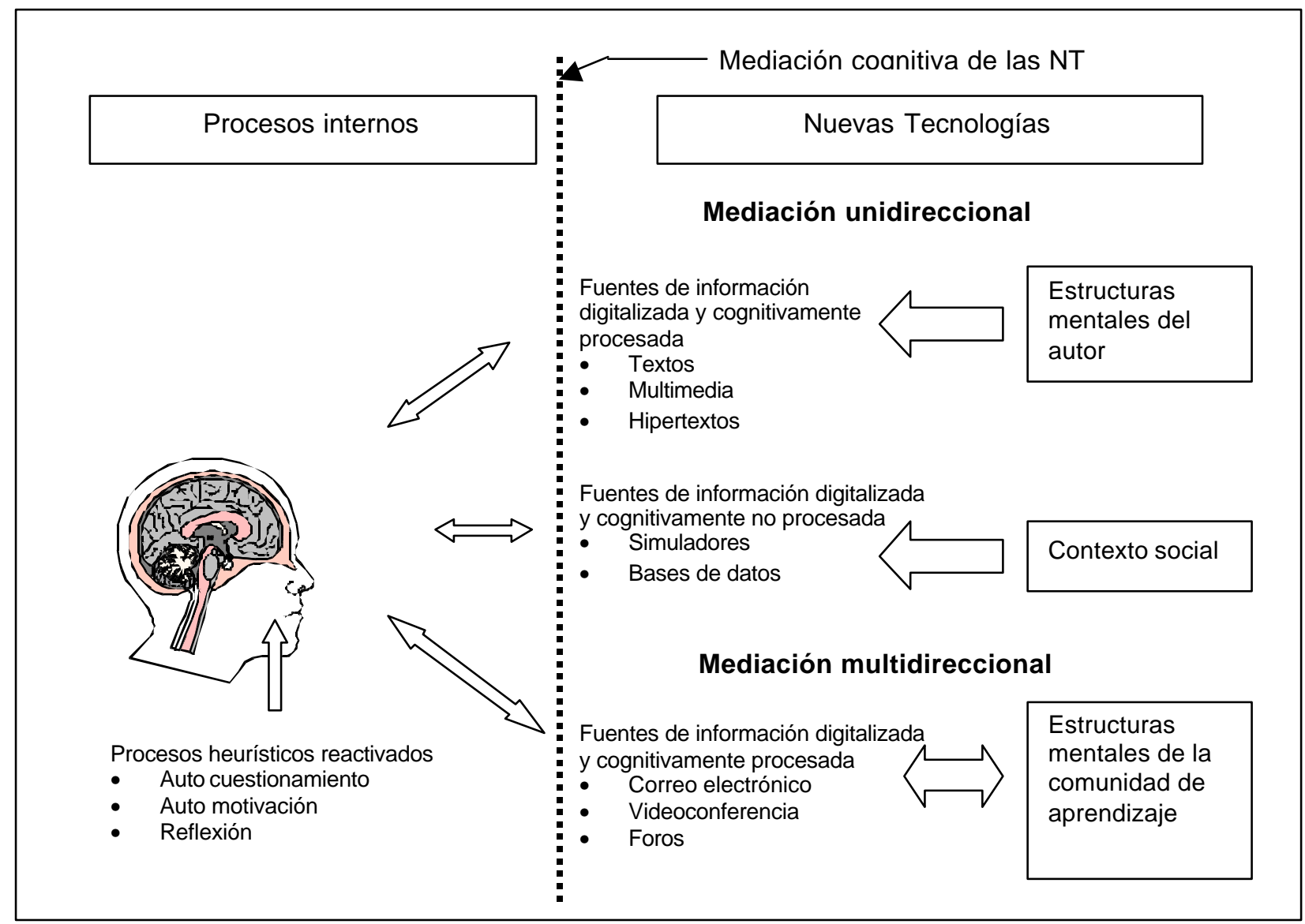

Figura 2: Mediación cognitiva de las NT

\subsection{Cinco estrategias didácticas surgidas del papel de las NT en el aprendizaje}

De acuerdo a las funciones cognitivas de las NT en el aprendizaje, es posible establecer cinco estrategias didácticas que permitirán desarrollar ambientes virtuales más propicios para el aprendizaje constructivo. Las técnicas y estrategias didácticas tienen como objetivo facilitar la adquisición de conocimientos, el desarrollo de habilidades y la generación o fortalecimiento de actitudes y valores, sin embargo, entre técnicas y estrategias existe una diferencia sustancial en sus alcances.

Una estrategia didáctica es una línea de acción que orienta y coordina un conjunto de actividades hacia una meta de aprendizaje claramente establecida. Una estrategia se pone de manifiesto a través de técnicas y procedimientos que pueden variar de un caso a otro, pero que tienen la misma finalidad: lograr una meta perfectamente establecida a través de una estrategia didáctica. Así, una estrategia puede valerse de muchas técnicas aplicadas de manera individual o combinada.

Una técnica didáctica, en cambio, corresponde a una meta más específica y tiene un alcance más corto. Las técnicas didácticas son procedimientos organizados para la consecución de objetivos de aprendizaje y establecen una secuencia de acciones puntuales para obtener de manera eficaz metas educativas. Las técnicas didácticas establecen también actividades de aprendizaje.

Llegado a este punto y de acuerdo al la revisión de la temática abordada hasta aquí, 
consideramos que las estrategias didácticas pueden ser las siguientes:

1. Propiciar el desequilibrio cognitivo. Como se vio, el aprendizaje puede generarse a través de un desequilibrio cognitivo, esto es, la falta de congruencia entre las estructuras mentales del individuo y una realidad observada que no puede explicarse. Cuando este desequilibrio es inducido desde afuera, de manera intencionada, se convierte en una estrategia didáctica. Aunque se presente información novedosa o abundante, no será suficiente para producir el aprendizaje. El desequilibrio cognitivo genera una "necesidad" de aprendizaje, una búsqueda para satisfacer dicha necesidad.

2. Propiciar la interacción de alto nivel cognitivo. Vygotski resaltó la importancia del entorno social y el uso de mediadores culturales en el aprendizaje. La interacción de alto nivel cognitivo se refiere a un intercambio de opiniones que muestren un alto contenido de ideas sustentadas en lo que se sabe, se cree o se piensa. Cuando esta interacción es propiciada y coordinada, se convierte en una estrategia didáctica.

3. Promover el desarrollo de habilidades del pensamiento. La didáctica actual señala la importancia de propiciar pensamiento crítico, así como las habilidades para aprender. El análisis, la síntesis, el razonamiento analógico, la observación sistemática, el uso del pensamiento crítico (diferentes posturas con respecto a un tema específico), el pensamiento divergente (diferentes soluciones a un mismo problema), son habilidades del pensamiento que deben propiciarse. Una orientación didáctica de procesos más que de contenidos, permite un mejor desarrollo de habilidades para pensar. Lo importante de esta estrategia es que no requiere contenidos específicos, en cualquier asignatura puede utilizarse. La promoción del desarrollo de habilidades para pensar requiere, sin embargo, la formación del docente en este campo.

4. Administrar los recursos atencionales. Como lo vimos anteriormente, una de las funciones más importantes de las NT en el aprendizaje, es la provisión de estímulos sensoriales. En este sentido, es importante establecer lineamientos que permitan la administración de los recursos atencionales. La atención es un recurso muy limitado. Por otro lado, cuando el ambiente virtual se encuentra en línea, el curso entra en competencia con otros sitios en la Web por la atención del usuario. Un esquema inadecuado de navegación, un abuso en la cantidad de información que se transmite, la dificultad para leer o extraer información, pueden distraer al aprendiz y propiciar la pérdida de atención. La información dosificada, la variación de estímulos o canales perceptivos pueden ayudar a administrar la atención. Nótese que la estrategia se refiere a la administración de los recursos atencionales, asumiendo que la atención es un recurso limitado, no permanente ni constante.

5. Administrar los recursos motivacionales. Como ya se mencionó, la motivación es una predisposición al aprendizaje que puede influir positiva o negativamente. En el caso de los ambientes virtuales de aprendizaje, existen muchos recursos sensoriales que pueden ser utilizados, sin embargo, la motivación, al igual que la atención son recursos limitados, es decir, no son permanentes, por lo que debe haber una buena administración de éstos 
recursos. Por ejemplo, si se elige la variación de estímulo como única técnica de motivación y se hace constantemente, la técnica pierde sentido y puede convertirse en un distractor.

Dichas estrategias surgen a partir del análisis realizado en torno a:

a) La generación del aprendizaje y la mediación cognitiva de las NT.

b)El papel de las NT y la provisión de estímulos sensoriales.

En el cuadro siguiente se presentan las cinco estrategias propuestas, así como la función de las NT en cada caso, además de ejemplos sobre los medios electrónicos y las técnicas didácticas que pueden usarse. Cabe aclarar que los medios y técnicas didácticas presentadas no pretenden ser exhaustivos sólo ilustrativos por lo que es evidente que existen muchas otras posibilidades en cada caso. 


\begin{tabular}{|c|c|c|c|c|c|}
\hline Estrategia & $\begin{array}{c}\text { Función de } \\
\text { las NT }\end{array}$ & Relación & Medio electrónico & Ejemplo de acciones & Técnica didáctica \\
\hline \multirow{3}{*}{$\begin{array}{l}\text { 1. Propiciar el } \\
\text { desequilibrio } \\
\text { cognitivo }\end{array}$} & \multirow{3}{*}{$\begin{array}{l}\text { Mediación } \\
\text { cognitiva }\end{array}$} & $\begin{array}{l}\text { Aprendiz- } \\
\text { fuentes de } \\
\text { información }\end{array}$ & $\begin{array}{ll}- & \text { Hipertexto } \\
- & \text { Multimedia }\end{array}$ & $\begin{array}{ll}- & \text { Preguntas } \\
& \text { retadoras } \\
\text { - } & \text { Aseveraciones } \\
\text { desafiantes }\end{array}$ & $\begin{array}{ll} & \text { Exposición } \\
\text { - } & \text { Aprendizaje } \\
& \text { basado en } \\
& \text { problemas, casos } \\
& \text { o proyectos. }\end{array}$ \\
\hline & & $\begin{array}{l}\text { Aprendiz- } \\
\text { contexto virtual }\end{array}$ & - $\quad$ Realidad virtual & $\begin{array}{ll} & \text { Escenarios } \\
& \text { virtuales } \\
& \text { retadores }\end{array}$ & $\begin{array}{l}\text { - Juego de roles } \\
\text { - Aprendizaje a } \\
\text { través de la } \\
\text { acción } \\
\text { Aprendizaje por } \\
\text { imitación }\end{array}$ \\
\hline & & $\begin{array}{l}\text { Aprendiz- } \\
\text { comunidad de } \\
\text { aprendizaje }\end{array}$ & \begin{tabular}{|ll}
- & Correo \\
& electrónico \\
- & Cursos en línea \\
- & Foros de \\
& discusión \\
\end{tabular} & $\begin{array}{ll}- & \text { Plantear } \\
& \text { preguntas } \\
\text { - Aseveraciones } \\
\text { desafiantes }\end{array}$ & $\begin{array}{ll} & \text { Aprendizaje } \\
\text { basado en } \\
\text { problemas, casos } \\
\text { o proyectos. }\end{array}$ \\
\hline \multirow{3}{*}{$\begin{array}{l}\text { 2. Propiciar la } \\
\text { interacción de } \\
\text { alto nivel } \\
\text { cognitivo }\end{array}$} & \multirow{3}{*}{$\begin{array}{l}\text { Mediación } \\
\text { cognitiva }\end{array}$} & Uno-uno & $\begin{array}{ll}- & \text { Correo } \\
& \text { electrónico } \\
\text { - } & \text { Hipertexto }\end{array}$ & $\begin{array}{ll} & \text { Ensayo con } \\
& \text { aportación } \\
& \text { personal } \\
\text { - Opinión } \\
\text { sustentada }\end{array}$ & $\begin{array}{ll} & \text { Exposición } \\
- & \text { Niveles de lectura } \\
& \text { Mapas } \\
& \text { conceptuales } \\
\end{array}$ \\
\hline & & Uno-muchos & 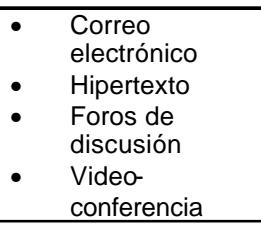 & $\begin{array}{ll} & \text { Sesiones de } \\
\text { preguntas y } \\
\text { respuestas } \\
\text { Confrontación } \\
\text { de opiniones } \\
\text { opuestas sobre } \\
\text { un tema } \\
\end{array}$ & $\begin{array}{ll} & \text { Exposición } \\
- & \text { Aprendizaje } \\
& \text { colaborativo }\end{array}$ \\
\hline & & $\begin{array}{l}\text { Muchos- } \\
\text { muchos }\end{array}$ & $\begin{array}{ll} & \text { Foros de } \\
& \text { discusión }\end{array}$ & $\begin{array}{ll} & \text { Debate } \\
& \text { sustentado }\end{array}$ & $\begin{array}{ll} & \text { Aprendizaje } \\
\text { colaborativo, } \\
\text { Aprendizaje } \\
\text { basado en } \\
\text { problemas, etc. }\end{array}$ \\
\hline \multirow[t]{2}{*}{$\begin{array}{l}\text { 3. Promover el } \\
\text { desarrollo de } \\
\text { habilidades } \\
\text { para pensar y } \\
\text { aprender }\end{array}$} & \multirow[t]{2}{*}{$\begin{array}{l}\text { Mediación } \\
\text { cognitiva }\end{array}$} & $\begin{array}{l}\text { Uno-uno } \\
\text { Uno-muchos }\end{array}$ & $\begin{array}{ll}- & \text { Hipertexto } \\
- & \text { Multimedia } \\
- & \text { Correo } \\
& \text { electrónico }\end{array}$ & $\begin{array}{ll} & \text { Discusión sobre } \\
\text { opiniones } \\
\text { opuestas en } \\
\text { torno a un tema }\end{array}$ & $\begin{array}{ll} & \text { Expansión y } \\
\text { compresión de } \\
\text { ideas } \\
\text { - } \quad \text { Herramientas } \\
\text { para pensar } \\
\text { - } \quad \text { Procesamiento } \\
\text { humano de la } \\
\text { información }\end{array}$ \\
\hline & & $\begin{array}{l}\text { Uno-consigo } \\
\text { mismo }\end{array}$ & $\begin{array}{ll}- & \text { Cursos WEB } \\
- & \text { Realidad virtual } \\
\text { - } & \text { Hipertexto } \\
- & \text { Multimedia } \\
\end{array}$ & $\begin{array}{ll}- & \text { Confrontación } \\
\text { del aprendiz con } \\
\text { su conocimiento } \\
\text { previo }\end{array}$ & $\begin{array}{ll} & \text { Mapas } \\
\text { - } & \text { conceptuales } \\
\text { - } & \text { Pretest-postest } \\
& \text { Metaconocimiento }\end{array}$ \\
\hline $\begin{array}{l}\text { 4. Administrar } \\
\text { los recursos } \\
\text { atencionales }\end{array}$ & & $\begin{array}{l}\text { Diseñador- } \\
\text { usuario }\end{array}$ & $\begin{array}{ll} & \text { Interfaz gráfica }\end{array}$ & $\begin{array}{ll}- & \text { Dosificación de } \\
& \text { la información } \\
\text { - } & \text { Destacar } \\
\text { información } \\
\text { relevante } \\
\text { - Inhibir estímulos } \\
\text { no deseados }\end{array}$ & \\
\hline $\begin{array}{l}\text { 5. Administrar } \\
\text { los recursos } \\
\text { motivacionales }\end{array}$ & $\begin{array}{l}\text { Provisión de } \\
\text { estímulos } \\
\text { sensoriales }\end{array}$ & $\begin{array}{l}\text { Diseñador- } \\
\text { usuario }\end{array}$ & - $\quad$ Interfaz gráfica & $\begin{array}{ll}-\quad & \text { Manejo de color, } \\
\text { imagen, } \\
\text { recursos } \\
\text { ambientales } \\
\text { Manejo } \\
\text { adecuado de la } \\
\text { información }\end{array}$ & \\
\hline
\end{tabular}

Figura 17: Relación entre las cinco estrategias propuestas, el papel de las NT en el aprendizaje y las técnicas didácticas 


\section{APLICACIÓN DE LAS ESTRATEGIAS EN UN AMBIENTE VIRTUAL}

Con base a las estrategias didácticas propuestas se desarrolló un ambiente virtual en el que interactuó una muestra aleatoria de estudiantes del segundo trimestre de la Universidad Autónoma Metropolitana. Los 33 estudiantes de la muestra realizaron dos actividades de aprendizaje diseñadas para propiciar el desequilibrio cognitivo y el desarrollo de habilidades del pensamiento. El diseño experimental o cuasiexperimental consistió en la aplicación de un pre-test y un pos-test. Entre uno y otro se desarrollaron las actividades de aprendizaje. Los resultados mostraron un avance significativo en los puntajes obtenidos antes y después de interactuar con el ambiente virtual de aprendizaje. En términos relativos se puede resumir los resultados en la siguiente tabla:

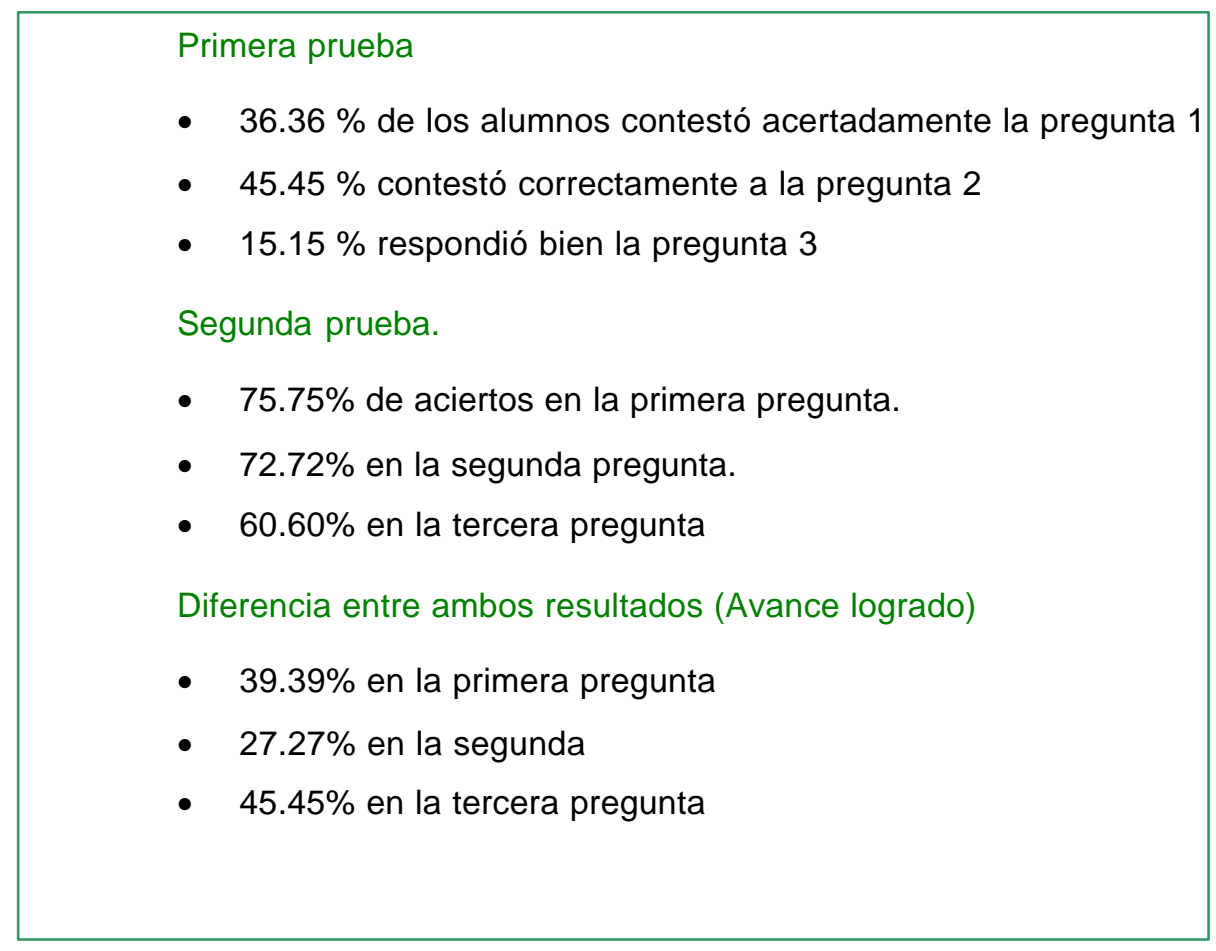

Porcentaje de alumnos que contestaron acertadamente las preguntas en el pretest y postest en la actividad 1

\section{Primera prueba}

- $\quad 15.15 \%$ de los alumnos contestó acertadamente la pregunta 1

- $\quad 21.21 \%$ contestó correctamente a la pregunta 2

- $\quad 42.42 \%$ respondió bien la pregunta 3

Segunda prueba.

- $\quad 48.48 \%$ de aciertos en la primera pregunta.

- $\quad 66.66 \%$ en la segunda pregunta.

- $\quad 69.69 \%$ en la tercera pregunta

Diferencia entre ambos resultados (Avance logrado)

- $\quad 33.33 \%$ en la primera pregunta

- $45.45 \%$ en la segunda

- $\quad 27.27 \%$ en la tercera pregunta 
Porcentaje de alumnos que contestaron acertadamente las preguntas en el pretest y postest en la actividad 2

\section{CONCLUSIONES}

El ejercicio de análisis y reflexión como el realizado en la investigación nos condujo a obtener una serie de conclusiones. Algunas de éstas surgen de la acción reflexiva del marco teórico y algunas más de la observación experimental.

- La revisión y el análisis de la literatura especializada nos permitió proponer dos funciones fundamentales de las nuevas tecnologías en el aprendizaje: la mediación cognitiva y la provisión de estímulos sensoriales.

- A partir de dichas funciones, se desarrolló un modelo instruccional sustentado en las teorías cognitivas que sirve de guía para el diseño de ambientes virtuales de aprendizaje.

- Las NT constituyen medios cada vez más valorados en la educación. La posibilidad de integrar los avances científicos en materia de aprendizaje, con los recursos tecnológicos ofrece alternativas que no pueden ignorarse, sin embargo, esta vinculación no se logra por "generación espontánea", es necesario crearla de manera intencionada y bajo conocimiento de causa.

- El uso adecuado de estrategias didácticas sustentadas en la psicología cognitiva como son:

a) Propiciar del desequilibrio cognitivo

b) Propiciar la interacción de alto nivel

c) Promover el desarrollo de habilidades para pensar y aprender

d) Administrar los recursos atencionales

e) Administrar los recursos motivacionales.

pueden favorecer el aprendizaje.

- Los resultados obtenidos en el pretest en ambas actividades mostraron un bajo rendimiento de los alumnos (3.23 y 4.72 sobre 10 respectivamente) a pesar de que las pruebas demandaban procesos básicos del pensamiento como: identificar, comparar y relacionar, lo cual muestra la necesidad de implementar programas educativos que permitan desarrollar habilidades para pensar. Esto confirma la preocupación de diversos investigadores (Sánchez 1993, Costa, 1991, Garza, 1998, entre otros) quienes han manifestado esta necesidad.

- Después de la aplicación de las estrategias didácticas, los alumnos parecieron comprender mejor los conceptos estudiados y pudieron obtener puntuaciones más altas que en la primera ocasión (6.97 y 7.78 en el postest correspondiente a la primera y 
segunda actividad respectivamente). Aunque existe un evidente avance (las puntuaciones se incrementaron el $43 \%$ y el $60 \%$ en el postest con respecto al pretest en cada una de las actividades), los resultados no son los ideales, si lo que se pretende es elevar la calidad y el nivel académico en nuestra universidad.

- El uso de estrategias didácticas respaldadas en las teorías cognitivas parecen favorecer el aprendizaje y el rendimiento académico de los alumnos. Los resultados obtenidos son congruentes con la hipótesis planteada. Sin embargo, debemos admitir que solamente a través de la evaluación continua y la investigación permanente será posible confirmar lo observado en este trabajo.

\section{BIBLIOGRAFÍA}

ANGLIN, G., (1995) Instructional Technology: Past, Present, and Future, Ed. Libraries

Unlimited, Inc., Colorado.

Arancibia, V., Herrera P., Strasser K., (1999) Psicología de la educación, Ed. Alfaomega, México.

ARAúJO, J., Chadwick, C., (1993) Tecnología educacional: Teorías de instrucción, Ed. Paidós, Ecuador.

BADILLO, S., (2003) "Diseño experimental de un ambiente de aprendizaje virtual:

una experiencia de investigación acción para el desarrollo de programas de postgrado en diseño". Documento desarrollado como reporte para el Área de Investigación de Nuevas Tecnologías, División de Ciencias y Artes para el Diseño, Universidad Autónoma Metropolitana, México.

BetancouRT, J., (2001) "Sistema didáctico interactivo para la enseñanza de los mecanismos en diseño industrial". Tesis para optar por el grado de Doctor en Diseño en la Línea de Nuevas Tecnologías, Universidad Autónoma Metropolitana, México.

BRIGGS, L., WAGER, W., (1981) Handbook of procedures for the design of instruction, Ed. Technology Publications, New Jersey.

BuITRÓN, M., (2003) "Consideraciones para el diseño de interfaces gráficas de usuario en ambientes virtuales educativos”. Tesis de Maestría. Tesis para optar por el grado de Maestra en Diseño en la Línea de Nuevas Tecnologías, Universidad Autónoma Metropolitana, Azcapotzalco, septiembre, México.

CABERO, J., Salinas, J., Duarte, A., Domingo, J., (2000) Nuevas tecnologías aplicadas a la educación, Ed. Síntesis, Madrid.

CAMPBELL, D., Stanley, J., (2001) Diseños experimentales y cuasiexperimentales en la investigación social, Ed.Amorrortu, Buenos Aires.

CAStAÑEDA, S., López, M., (1995) “Manual para el curso de Psicología de la Educación”, Ed. ITESM, México. 
CAStAÑEdA, S., Almaguer, M., (1993) "Manual para el curso de Psicología cognitiva", Ed. ITESM, México.

COSTA, A., (1991) Developing Minds: Programs for Teaching Thinking, Ed.Association for Supervision and Curriculum Development, Alexandria.

DE VEGA, M., (1993) Introducción a la psicología cognitiva, Ed. Alianza, Madrid.

FAINHOLC, B., (1999) La interactividad en la educación a distancia, Ed. Paidós, Argentina.

FerReIRO, E., (1999) Vigencia de Jean Piaget, Ed. Siglo XXI, México.

GARZA, R., Leventhal S., (1998) Aprender cómo aprender. Ed. Trillas,México.

GAGNÉ, R., (1985) The conditions of Learning. Ed. Holt, Rinehart \& Winston, New York.

GAGNÉ, R., Briggs, L. (1994) La planificación de la enseñanza: Sus principios, Ed. Trillas, México.

GONZÁLEZ, O., Flores, M., (2000) El trabajo docente: enfoques innovadores para el diseño de un curso. Ed.Trillas, México.

GONZÁLEZ, M., (2003) "Hipertexto y aprendizaje", Anuario 2003, Área de Investigación de Nuevas Tecnologías. pp (9-15), Ed. por la Universidad Autónoma Metropolitana-Azcapotzalco, Ciencias y Artes para el Diseño, México.

HeRRERA, M., (2002) "Las fuentes del aprendizaje en ambientes virtuales educativos", Revista Iberoamericana de Educación, ISSN: 1681-5653, sep. Versión disponible en formato PDF en http://www.campus-oei.org/revista/index/frame_novedades.htm

HerRerA, M., (2003) "Las fuentes del aprendizaje en ambientes virtuales educativos" Anuario 2003, Área de Investigación de Nuevas Tecnologías, (pp 28-34), Ed. por la Universidad Autónoma Metropolitana-Azcapotzalco, Ciencias y Artes para el Diseño, México

KHAN, B., et al., (1998) Web-Based Instruction Ed. Educational Technology Publications, Englewood Cliffs, New Jersey.

MiAlARET, G., (2001) Psicología de la educación. Ed. Siglo XXI, México.

MOREIRA, H., (1998) "Hacia un nuevo modelo del proceso de enseñanza-aprendizaje basado en la Misión del Tecnológico de Monterrey para el año 2005", Documento de trabajo Tercera Versión, Abril, Monterrey.

Nemirovski, G., Neuhaus, U., Schlageter, G., (1998)"Setting Requirements for Learning Software", Ed-Media \& Ed-Telecom '98, Freiburg, Germany, June.

NovAK, J., Gowin, B., (1987) Aprendiendo a aprender, Ed. Martínez Roca, Barcelona.PozO, J., (1994) Teorías cognitivas del aprendizaje, Ed. Morata, Madrid.

ROGERS, P., (2002) Designing Instruction for Technology-Enhanced Learning, Ed. Idea Group Publishing, Hershey, U.S.A. 
SÁnCHEZ, M., (1993) Desarrollo de habilidades del pensamiento: Procesos Básicos del Pensamiento, Guía del Instructor, Ed. Trillas, México.

WoOlfolk, A., (1990) Psicología educativa, Ed. Prentice Hall, México.

Woolfolk, A., (1995) Educational Psychology, Ed. Allyn \& Bacon, Ohio State. 


\title{
Contactar
}

Revista lberoamericana de Educación

\author{
Principal OEI
}

